\title{
Effect of functional bracing, quadriceps and hamstrings on anterior tibial translation in anterior cruciate ligament insufficiency: A preliminary study
}

Jens Bagger, MD; Jesper Ravn, MD; Peter Lavard, MD; Peter Blyme, MD; Christian Sørensen, MD Department of Orthopaedics T, Gentofte Hospital, Copenhagen, Denmark

\begin{abstract}
Anterior tibial translation was measured in six patients with anterior cruciate ligament insufficiency. The tests were done in 15, 45, and 90 degrees of knee flexion, partly with activated quadriceps or hamstrings, and partly with subjects wearing a 4-point functional DonJoy brace. The translation was evaluated with a computerized electrogoniometer (Acufex KSS). The anterior tibial translation was significantly reduced by use of the hamstrings in all three degrees of knee flexion. The effect of the 4-point brace was only significant in 15 and 45 degrees of flexion. The quadriceps did not reduce the anterior tibial translation.
\end{abstract}

Key words: Acufex KSS, anterior cruciate ligament insufficiency, anterior tibial translation, functional DonJoy brace, hamstrings, knee flexion, quadriceps.

\section{INTRODUCTION}

The treatment of anterior cruciate ligament (ACL) insufficiency is still controversial $(1,2,3)$, and many aspects concerning the biomechanics of the knee still need to be investigated (4). A major component in patients with $\mathrm{ACL}$ insufficiency is the anterior tibial translation (ATT) in relation to the femur. If conservative treatment is chosen, or if operative treatment is followed by early training, a functional brace can benefit the patient $(5,6,7)$.

\footnotetext{
Address all correspondence and requests for reprints to: Jens Bagger, MD, Department of Orthopaedics T, Gentofte Hospital, Niels Andersens vej 65, DK 2900 Hallerup, Denmark.
}

The purpose of this study is to test the effect of the extensors and the flexors of the knee, as well as the effect of a functional brace, on the ATT in patients with ACL insufficiency.

\section{METHOD}

Six patients with unilateral $\mathrm{ACL}$ injuries were included in the study. (For patient characteristics see Table 1.) All patients had daily subjective complaints because of ACL insufficiency.

In order to measure the ATT, the ACL-insufficient knees were tested with an Acufex KSS laxity tester, using the contralateral knee as the control. Translation force was $100 \mathrm{~N}$. The Acufex KSS (Figure 1 and Figure 2) is a computerized electrogoniometer consisting of a test device mounted on the knee. Four potentiometers registered the anteroposterior tibial translation, knee flexion, tibial rotation, and varus/valgus displacement of the tibia in relation to the femur. The results were displayed on a computer monitor. With a special device connected to the computer, the anteroposterior translation force was applied to the tibia and also displayed on the monitor. The tests were done under standardized conditions because the same degree of knee flexion and the same applied force was repeated in each test. Each test was performed at least twice to be sure that the patient cooperated sufficiently. During each test, the patient sat on a test table with the leg clasped on a stand. Tests were done in three fixed degrees of knee flexion: 15 , 


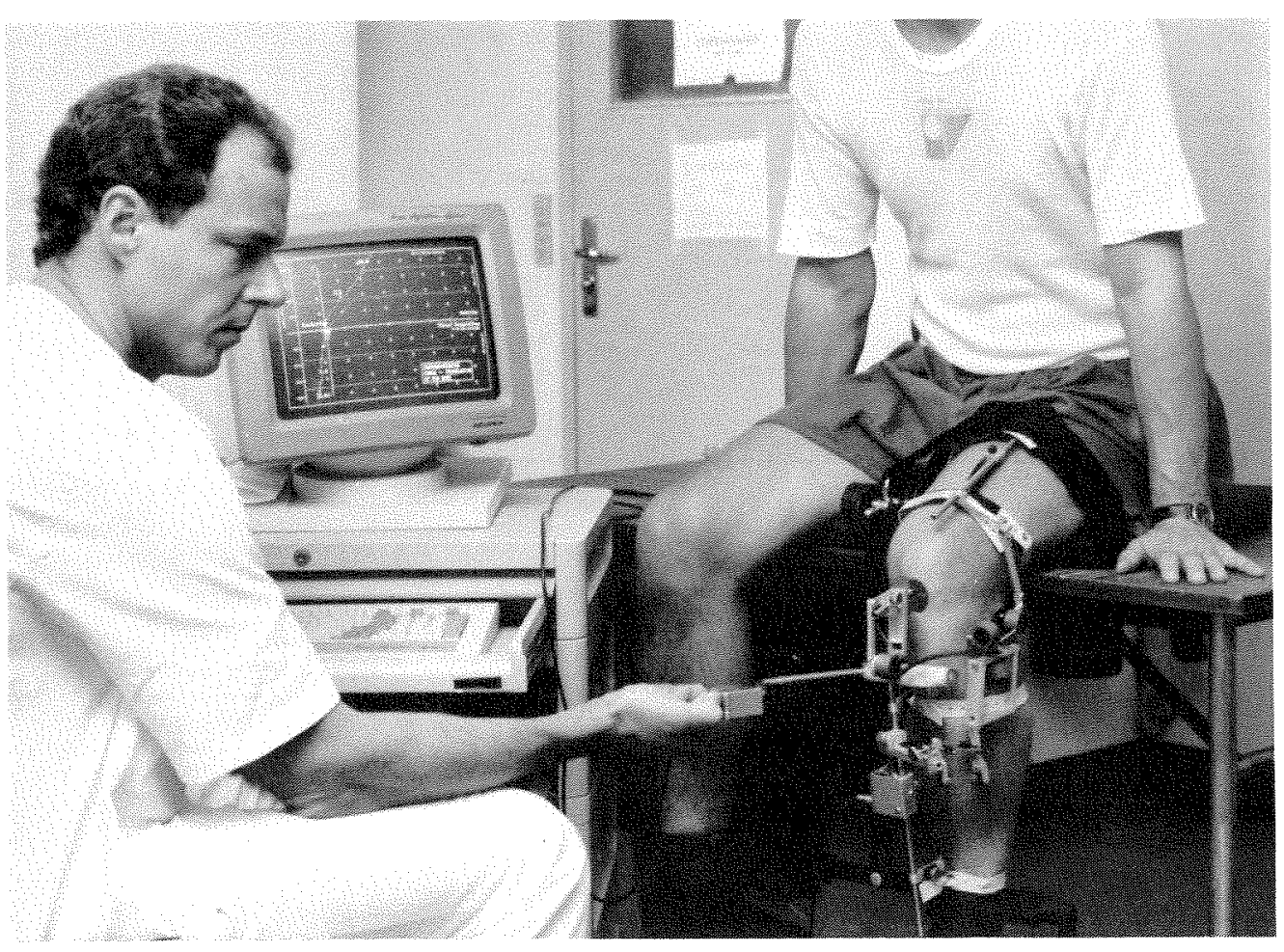

Figure 1.

The Acufex KSS: test setup.
45, and 90 degrees. Between each test, the patient rested for 1 to 2 minutes. Each test took 3 to 5 seconds.

The tests were done in four phases. During Phase 1, the ATT was measured on the relaxed knee, comparing

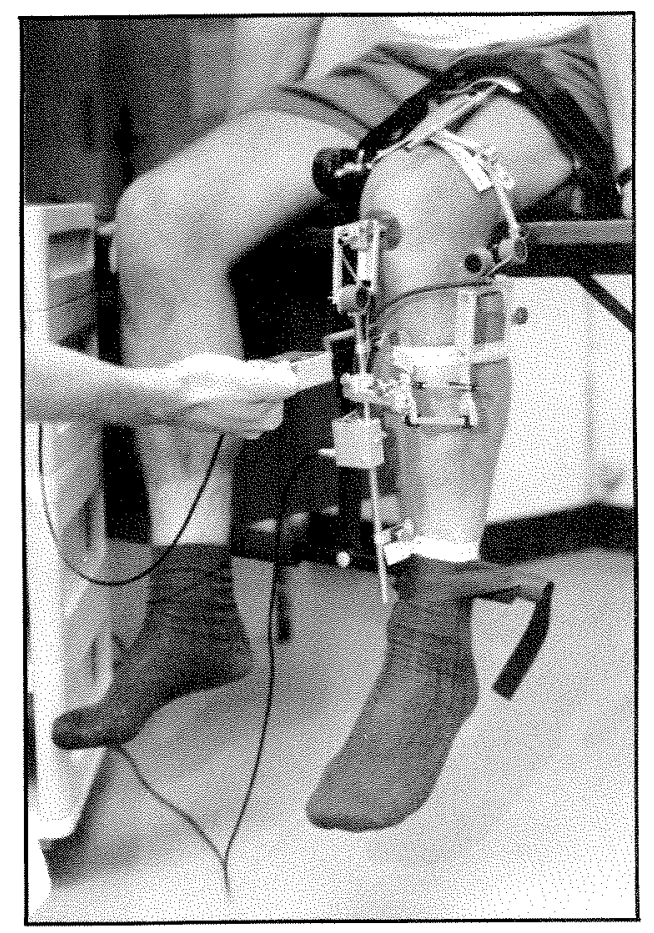

Figure 2.

The Acufex KSS: test device mounted on the knee. the ACL-insufficient knee to the normal knee. The other phases tested only the ACL-insufficient knee. In Phase 2, the ATT was measured while the patients used their maximal hamstring force, and in Phase 3, they used maximal quadriceps force. In Phase 4, the ATT was measured on the relaxed knee with the knee braced with a 4-point functional brace (DonJoy-4-P).

The brace (Figure 3) consisted of two vertical aluminum alloy bars on each side of the knee, hinged by adjustable flexion-extension stops. Two aluminum alloy cuffs, an anterior thigh cuff, and a posterior calf cuff were fixed to the bars. The 4-point support was secured by two straps-a posterior thigh strap, and an anterior tibial strap; both were placed closer to the knee joint than the two cuffs. Before the trials, 180 measurements were completed on

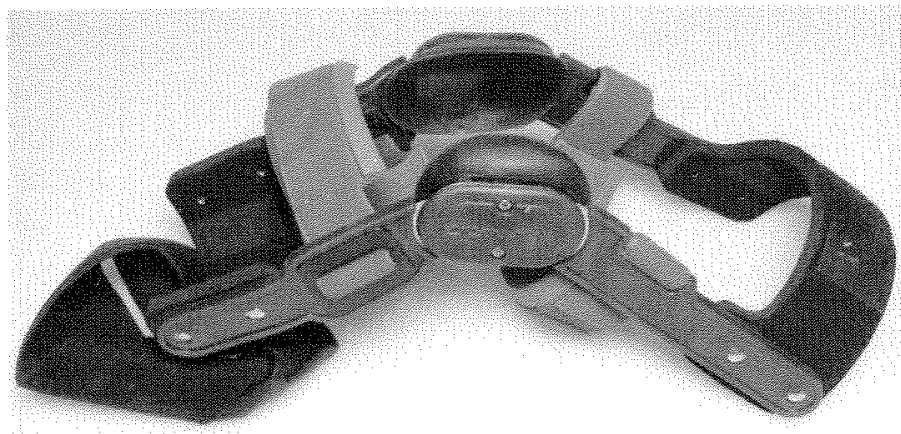

Figure 3.

The functional brace: DonJoy-4-P. 
Table 1.

Patient characteristics.

\begin{tabular}{|c|c|c|c|c|c|c|}
\hline Patient No. & 1 & 2 & 3 & 4 & 5 & 6 \\
\hline sex & F & M & $\mathrm{F}$ & M & M & M \\
\hline diagnosis & arthrotomy & arthroscopy & arthroscopy & arthroscopy & arthroscopy & arthroscopy \\
\hline treatment (ACL) & suture & none & none & none & none & none \\
\hline injury/study (time) & $7 \mathrm{~m}$ & $8 \mathrm{~m}$ & $60 \mathrm{~m}$ & $6 \mathrm{~m}$ & $5 \mathrm{~m}$ & $1 \mathrm{~m}$ \\
\hline
\end{tabular}

6 normal knees to verify the accuracy of the test apparatus. If the test device was not removed from the knee during the tests, 92 percent of the tests were equal to, or differed from, the maximum of $\pm 1 \mathrm{~mm}$ from the median value. If the test device was removed from the knee between the tests, the accuracy was 78 percent $(p<0.05)$.

As the leg was fixed in the test stand, varus/valgus displacement was zero, and in the tests of the ACLinsufficient knees, tibial rotation varied from zero to 13 degrees, with a median value of 1 degree. It was therefore assumed that the measurements of the tibial translation was not influenced by either varus/valgus displacement or tibial rotation in this setup.

\section{RESULTS}

Average ATT of the ACL-insufficient knee was compared with the nonaffected knee (see Table 2). The ATT was significantly demonstrated only in 15 degrees of knee flexion. A stabilizing effect on the ATT was obtained using the hamstrings (Table 3). The hamstrings significantly reduced the ATT as compared to the relaxed knee. The reduction, when the knee was fixed in 15 degrees of flexion, was 88 percent $(\mathrm{p}<0.01)$; 94 percent in 45 degrees of flexion $(p<0.025)$; and 81 percent in 90 degrees of flexion $(\mathrm{p}<0.01)$.

In Phase 3, the measurements were done while the quadriceps was contracted and seemed to show a stabilizing effect on the ATT. But at the end of each test the patients relaxed their quadriceps, resulting in a backward gliding of the tibia. The sum of the measured reduction in ATT and the backward gliding was approximately equal to the primary measured ATT on the relaxed knee.

Table 3 shows that the 4-point brace significantly reduced the average ATT as compared to the relaxed knee in both $15(\mathrm{p}<0.05)$ and $45(\mathrm{p}<0.025)$ degrees of flexion, but not in 90 degrees of flexion $(p<0.25)$. There was no statistical difference in the stabilizing effect when comparing the hamstring force to the brace.

\section{DISCUSSION}

This study was done with each patient sitting on a table with the knee fixed. The tibial rotation and varus/valgus displacement was negligible with this setup. Therefore, the results represent a static analysis of the stabilizing effect

Table 2.

Anterior tibial translation in relation to degrees of flexion for Affected and Non-affected knees.

\begin{tabular}{lccccc}
\hline ATT/Degrees of flexion & $\begin{array}{c}\text { Average value }(\mathbf{m m}) \\
\text { Affected knee }\end{array}$ & Range & $\begin{array}{c}\text { Average value }(\mathbf{m m}) \\
\text { Non-affected knee }\end{array}$ & \multicolumn{1}{c}{ Range } \\
\hline 15 & 7.8 & $(12.3-3.6)$ & 3.3 & $(6.8-0.3)$ & $\mathrm{p}<0.05$ \\
45 & 6.0 & $(12.7-3.8)$ & 5.3 & $(7.2-1.6)$ & $\mathrm{p}<0.25$ NS \\
90 & 2.0 & $(4.2-0.5)$ & 0.9 & $(2.2-0.0)$ & $\mathrm{p}<0.4$ NS \\
\hline
\end{tabular}

Statistics were obtained by paired Student's $t$-test and the level of significance was 95 percent. 
Journal of Rehabilitation Research and Development Vol. 29 No. 1 Winter 1992

Table 3.

ATT in ACL-insufficient knees in 15, 45, and 90 degrees of flexion for relaxed knee, using hamstring force, and with brace.

\begin{tabular}{|c|c|c|c|c|c|c|c|c|}
\hline Degrees of flexion & 1 & 2 & 3 & 4 & 5 & 6 & p value & $\begin{array}{l}\text { Average decrease of } \\
\text { ATT in percent }\end{array}$ \\
\hline 15 & 11.1 & 9.3 & 12.3 & 5.4 & 3.6 & 4.9 & & \\
\hline $15 \mathrm{D}$ & 1.7 & 4.7 & 2.4 & 3.0 & 0.7 & 4.0 & $\mathrm{p}<0.05$ & 64 \\
\hline $15 \mathrm{H}$ & 2.4 & 0.8 & 0.1 & 0.5 & 0.9 & 0.7 & $\mathrm{p}<0.01$ & 88 \\
\hline $45 \mathrm{D}$ & 0.4 & 1.9 & 1.7 & 0.7 & 1.6 & 0.9 & $\mathrm{p}<0.025$ & 82 \\
\hline $45 \mathrm{H}$ & 1.4 & 0.3 & 0.2 & 0.1 & 0.1 & 0.3 & $\mathrm{p}<0.025$ & 94 \\
\hline 90 & 4.2 & 3.2 & 2.6 & 1.7 & 0.5 & 1.8 & & \\
\hline $90 \mathrm{D}$ & - & 1.6 & 0.3 & 0.5 & 0.1 & 0.2 & $\mathrm{p}<0.25$ & 72 \\
\hline
\end{tabular}

D: with brace;

of knee extensors and flexors, as well as the effect of a 4-point functional brace. The reproducibility of the Acufex KSS electrogoniometer was acceptable. In the first phase of the study, the difference between ATT in the affected knee and the normal knee was clearly demonstrated in 15 degrees of flexion, replicating the clinical Lachmann test. When the patients used their maximal hamstring force, the ATT was significantly reduced, thus stabilizing the knee. The effect on the hamstrings was probably due to drawing the tibia backward while the posterior cruciate ligament acted as a counterstop, thereby stabilizing the knee (8). The quadriceps drew the tibia forward and at first had a stabilizing effect when maximally contracted. The lack of ACL led to a considerable sliding forward and backward of the tibia, resulting in a great load on the joint surface. Therefore, the quadriceps had no stabilizing effect on the ACL-insufficient knee. Conservative treatment for these patients should focus on hamstring exercises. The 4-point brace seemed to fixate the tibia in relation to the femur, avoiding inexpedient sliding of the tibia (9), but this needs to be further investigated in dynamic tests. As the healing time for reconstructed or augmented ACL may last up to one year (10), the use of a 4-point brace during active training might improve the long-term results for patients with ACL injuries.

\section{REFERENCES}

1. Eriksson E. Reconstruction of anterior cruciate ligament. Orthop Clin North Am 1977;7:167-179.

2. Jensen JE, Slocum DB, Larsson RL, et al. Reconstruction procedures for anterior cruciate ligament insufficiency: A computer analysis of clinical results. Am J Sports Med 1983;11:240-248.

3. Libscomb AB, Johnston RK, Snyder RB. The technique of cruciate ligament reconstruction. Am J Sports Med 1981;9:77-81.

4. Arms SW, Pope MH, Johnson RJ, et al. The biomechanics of anterior cruciate rehabilitation and reconstruction. Am J Sports Med 1984;12:8-18.

5. Beck C, Drez D, Young J, et al. Instrumented testing of functional braces. Am J Sports Med 1986;14:253-256.

6. Cawley PW. Functional bracing for skiing: A review of factors affecting brace choice. Top Acute Care Trauma Rehabil 1988; 3:73-81.

7. Tegner Y, Petterson G, Lysholm J, et al. The effect of derotation braces on knee motion. Acta Orthop Scand 1988;59:284-287.

8. Walla DJ, Albright JP, McAuley E, et al. Hamstring control and unstable ligament-deficient knee. Am J Sports Med 1985; 13:34-39.

9. Colville MR, Lee CL, Ciullo JV. The Lennox Hill brace. An evaluation of effectiveness in training knee instability. Am J Sports Med 1986;14:257-260.

10. Paulos L, Noyes FR, Grood E. Knee rehabilitation after anterior cruciate ligament reconstruction and repair. Am J Sports Med 1981;9:140-149. 